\title{
EDITORIAL
}

\section{¿Debemos acogernos a las nuevas recomendaciones de diagnóstico sobre hipertensión arterial?}

\section{Should we adhere to the new diagnostic recommendations for arterial hypertension?}

\author{
Dagnóvar Aristizábal-Ocampo
}

\author{
Centro Clínico y de Investigación SICOR, Medellín, Colombia
}

Recibido el 27 de diciembre de 2017; aceptado el 27 de diciembre de 2017

Disponible en Internet el 11 de enero de 2018

\begin{abstract}
La detección temprana de la enfermedad y la precisión diagnóstica tienen grandes beneficios para la salud pública sin embargo, estas ganancias tienen su precio. Con la obtención de más evidencias a partir de los ensayos clínicos, el desarrollo de nuevos métodos diagnósticos y mejoras tecnológicas en medicina, la línea entre lo normal y anormal se hace muy tenue y pequeños cambios fisiológicos pueden tornar nuestra aparente normalidad en anormalidad. Es este el escenario al cual hemos desembocado en hipertensión arterial a raíz de la publicación reciente de la guía conjunta del Colegio Americano de Cardiología y la Asociación Americana del Corazón (ACC/AHA por sus siglas en inglés $)^{1}$, la cual considera que una presión arterial (PA) sistólica $\geq 120 \mathrm{~mm}$ Hg está elevada y clasifica la hipertensión estadio I a partir de $130 / 80 \mathrm{~mm}$ Hg. Esta nueva clasificación contrasta con lo publicado en la última guía europea de hipertensión ${ }^{2}$ y en el denominado JNC8 ${ }^{3}$.

La hipertensión arterial es uno de los factores con mayor riesgo atribuible en poblaciones (RAP). En el estudio realizado en Medellín, Colombia, por nuestro grupo, se halló un RAP de enfermedad coronaria del $32 \%$ al tomar un punto de corte para hipertensión de $140 / 90 \mathrm{~mm} \mathrm{Hg}$, es decir que el $32 \%$ de eventos coronarios (infarto de miocardio, angina de pecho) se neutralizarían si la población en mención no fuera hipertensa ${ }^{4}$. Ahora, bajo los nuevos puntos de corte este
\end{abstract}

Correo electrónico: dagnovar@une.net.co valor sería del $40 \%$. Los investigadores de la Organización Mundial de la Salud han encontrado que presiones sistólicas de más de $115 \mathrm{~mm} \mathrm{Hg}$ o diastólica de más de $75 \mathrm{~mm} \mathrm{Hg}$ explican el $49 \%$ de la incidencia de eventos coronarios y el $62 \%$ de ataques cerebrovasculares ${ }^{5}$, dato que concuerda con lo observado en nuestra población.

Los beneficios de mantener una PA normal en términos de neutralizar riesgos cardiovasculares son innegables, lo cual, como bien sabemos, se puede lograr en la mayoría de individuos con medidas como mantener un peso adecuado, realizar actividad física frecuente, reducir la ingesta de sal y alcohol y aumentar el consumo de frutas y verduras ${ }^{1,6}$. Por tanto, a la pregunta de si ¿es una PA $<120 / 80 \mathrm{~mm} \mathrm{Hg}$ considerada normotensión? la respuesta es afirmativa y era algo que ya se tenía claro.

Ahora bien, el gran cambio con esta última guía está en proponer que la intervención terapéutica (no farmacológica) debe empezar en presiones mayores de $120 \mathrm{~mm} \mathrm{Hg}$ si el riesgo cardiovascular es $<10 \%$ a 10 años y si la presión sistólica es $130 \mathrm{~mm} \mathrm{Hg} \mathrm{y} \mathrm{este} \mathrm{riesgo} \mathrm{es} \mathrm{>} \mathrm{10 \% ,} \mathrm{se} \mathrm{debe} \mathrm{incluir}$ además tratamiento farmacológico; también se indica que, en individuos ya hipertensos, la PA debe reducirse no hasta $140 \mathrm{~mm} \mathrm{Hg}$ sino a valores $<130 \mathrm{~mm} \mathrm{Hg}$. Ante estas modificaciones, considero que se requiere determinar mucho mejor los criterios para definir en qué pacientes y con qué intensidad realizar el tratamiento y no solamente el punto al cual se pretende reducir la PA. Dicho de otra manera, hay que individualizar mucho más el manejo del paciente hipertenso. 
Desde que quedó claro que la PA alta tenía una relación directa con enfermedad cardiovascular, nos hemos preguntado si reducir la PA más que lo publicado en guías previas (por debajo de $140 \mathrm{~mm} \mathrm{Hg}$ ) confiere mayores beneficios cardiovasculares. Esto ha sido tema de investigación en las últimas tres décadas y los hallazgos hacen que lo enunciado por la reciente guía ACC 2017 no nos llegue por sorpresa. Con los estudios SPRINT ${ }^{7}$ y ACCORD ${ }^{8}$ son ya más de 20 ensayos clínicos con 54.000 pacientes incluidos que indican que un tratamiento más intenso de la hipertensión es mejor que uno menos intenso para varios grupos de alto riesgo?.

Los resultados del SPRINT ${ }^{7}$ están de acuerdo con algunos metaanálisis recientes que han incluido varios ensayos clínicos con un número grande de pacientes y desenlaces. Xie et al. ${ }^{10}$ compararon el efecto del tratamiento más intensivo versus menos intensivo para reducir la presión y los efectos sobre desenlaces cardiovasculares y renales. Los hallazgos muestran que el tratamiento intensivo logró una presión sistólica más baja de $133 \mathrm{~mm} \mathrm{Hg}$ comparado con $140 \mathrm{~mm} \mathrm{Hg}$ en el grupo de tratamiento menos intenso. Esta diferencia se acompañó de una reducción significativa en el riesgo de eventos cardiovasculares mayores, infarto de miocardio y ataque cerebrovascular (reducción en riesgo relativo del $15-20 \%$ ) y en pacientes diabéticos de la albuminuria o progresión de la retinopatía. Estos efectos benéficos deben ser contrastados con el número de efectos secundarios adversos que se han asociado regularmente con el tratamiento llevado a presiones más bajas. En el SPRINT los pacientes tratados con meta de presión sistólica de $120 \mathrm{~mm}$ Hg tuvieron más hipotensión, síncope, trastornos electrolíticos, así como lesión y falla renal.

Si bien estos ensayos apoyan un cambio en la meta de reducción de la PA con el tratamiento antihipertensivo, también indican que debe elegirse bien en quien y como debe hacerse esta reducción de la PA. Como el efecto terapéutico es diferencial dependiendo del nivel de riesgo cardiovascular y tipo de paciente considerado (viejo, diabético, hipertenso sistólico, con enfermedad renal, etc.), se necesitan más estudios en los que se haga una mejor caracterización de los hipertensos por sus particularidades fenotípicas, otros marcadores de riesgo y comorbilidades para definir el tipo de tratamiento a seleccionar ${ }^{11}$. De nuevo, este es un llamado a individualizar el tratamiento según nivel de riesgo y comorbilidades.

\section{La elección del tratamiento debe tener en cuenta el tipo de paciente y riesgo cardiovascular cuantificado}

No todos los antihipertensivos ni tipos de combinaciones farmacológicas reducen la PA en la misma forma, ni la protección cardiovascular se logra en todo hipertenso con obtener presiones arteriales más bajas, si no se tienen en cuenta los efectos adversos potenciales. Esto claramente lleva a que la categorización del riesgo sea un factor central en la toma de decisión para definir la intensidad del tratamiento farmacológico y la meta de tratamiento en términos de PA. La reciente guía ACC 2017 lo anuncia al decir: "para cierta cantidad de reducción de la PA con antihipertensivos, un menor número de individuos en alto riesgo necesitan ser tratados para prevenir un evento cardiovascular, en comparación con individuos en bajo riesgo"'. A manera de ejemplo un paciente diabético o con enfermedad renal crónica requiere una estrategia más intensiva de tratamiento que un paciente joven o quien no presenta otros riesgos identificados. Igualmente se deben incluir en este grupo de mayor riesgo los pacientes con rigidez arterial o riesgo de aterosclerosis a 10 años $\geq 10 \%$. Igualmente hacen parte de este grupo los pacientes hipertensos con enfermedad coronaria que requieren prevención secundaria y en quienes lograr una PA de $120 \mathrm{~mm} \mathrm{Hg}$ frecuentemente estaría acompañado de una reducción excesiva de la presión diastólica. Es en estos pacientes en quienes la combinación de las medidas no farmacológicas más el tratamiento farmacológico deben aplicarse en forma combinada, efectiva y con destreza. Y debo enfatizar en forma efectiva ya que la prescripción del tratamiento farmacológico y no farmacológico por el clínico, es solo una parte del plan que termine en beneficios para el paciente. La ejecución correcta de este plan terapéutico requiere educación y acompañamiento al paciente por parte de un equipo interdisciplinario.

\section{Consideraciones importantes para implementar un tratamiento antihipertensivo con metas más estrictas}

Aceptando la evidencia que muestra que una meta más estricta de PA proporciona mejor protección cardiovascular, una vez individualizado el tratamiento para mitigar los efectos adversos potenciales, se deben tener en cuenta los siguientes elementos:

1. El beneficio potencial vs. los efectos adversos del tratamiento intensivo: resulta muy importante establecer cuál es el nivel de riesgo y comorbilidades a intervenir y tener presente si la meta terapéutica no genera riesgos que afectan el beneficio esperado. Si el riesgo cardiovascular es mayor de 10\% a 10 años, la intervención para llevar al paciente a una meta de $130 \mathrm{~mm} \mathrm{Hg}$ o menor requerirá un manejo farmacológico intenso con hasta tres o más medicamentos, para lo cual hay que tener presente que: a) Los antihipertensivos tienen efectos aditivos y en general manejar dosis más bajas y agregar una nueva clase de antihipertensivo (terapia de combinación) logra mayor descenso de la PA que doblar la dosis de un solo fármaco, esto cuando se utilizan tiazida, betabloqueador, antagonistas de receptor de angiotensina II y calcioantagonista ${ }^{12}$; b) Lograr una presión sistólica de $120-130 \mathrm{~mm} \mathrm{Hg}$ en la mayoría de pacientes > 75 se acompañará frecuentemente de una presión diastólica < $70 \mathrm{~mm} \mathrm{Hg}$ y esto puede comprometer el flujo coronario y de otros territorios debido a la conocida curva $\mathrm{J}$ de la presión diastólica ${ }^{13}$.

2. La forma como los medicamentos interactúan en combinaciones debe entenderse bien ante la necesidad de usar de dos o tres antihipertensivos para llegar a esas metas. Este aspecto es aún más importante por los efectos que la reducción intensa de la PA tiene sobre la función renal. Tanto en el SPRINT como en el ACCORD, la mayor reducción en la PA se acompañó de un aumento en los niveles de creatinina y aun falla renal aguda. Esto, junto con las alteraciones en electrolitos más comunes en este grupo 
intensamente tratado, significa un evento de lesión o falla renal aguda por cada 63 pacientes tratados, un paciente llevado a hipotensión por cada 99 tratados y una alteración electrolítica por cada 128 tratados $^{14}$.

3. El nuevo rótulo de hipertenso a los individuos previamente normotensos: frente a la categorización de presión alta a partir de $120 \mathrm{~mm} \mathrm{Hg}$, cabe que los clínicos se pregunten: ¿cómo evitar que un individuo se sienta rotulado y enfermo sabiendo que se le ha dicho que tiene la presión alta? Esto constituye un reto en la implementación de este nuevo estándar que llevó a tener presión anormal a muchos que antes disfrutaban de ser normotensos. Ciertamente lo que se ha dado en llamar la "tiranía de la normalidad" tiene su consideración en este nuevo escenario.

4. Las consecuencias epidemiológicas y para el sistema de salud: he evitado referirme a los efectos de esta nueva clasificación sobre la salud pública en Colombia y otros países Latinoamericanos en términos del aumento del diagnóstico y tratamiento de la hipertensión, sin embargo, el tener $25 \%$ de nuevos hipertensos en la población acarreará consecuencias inevitables sobre nuestros sistemas de salud, la mayoría de ellos basados en el aseguramiento público y que aun con la prevalencia actual maneja un pobre control de la hipertensión en nuestras poblaciones. Los efectos de esta nueva "carga" de personas con presión alta y de hipertensos que requieren tratamientos intensivos impactarán fuertemente los recursos de nuestros sistemas de salud.

\section{Bibliografía}

1. Whelton PK, Carey RM, Aronow WS, Casey DE, Jr., Collins KJ, Dennison Himmelfarb C et al., 2017.ACC/AHA/AAPA/ ABC/ACPM/AGS/APhA/ASH/ASPC/NMA/PCNA Guideline for the Prevention, Detection, Evaluation, and Management of High Blood Pressure in Adults: A Report of the American College of Cardiology/American Heart Association Task Force on Clinical Practice Guidelines. J Am Coll Cardiol. 2017, 23976; https://doi.org/10.1161/HYP. 0000000000000066.

2. Mancia G, Fagard R, Narkiewicz K, Redon J, Zanchetti A, Bohm $\mathrm{M}$, et al. $2013 \mathrm{ESH} / \mathrm{ESC}$ guidelines for the management of arterial hypertension: the Task Force for the Management of Arterial Hypertension of the European Society of Hypertension (ESH) and of the European Society of Cardiology (ESC). Eur Heart J. 2013;34:2159-219.

3. James PA, Oparil S, Carter BL, Cushman WC, DennisonHimmelfarb C, Handler J, et al. 2014 evidence-based guideline for the management of high blood pressure in adults: report from the panel members appointed to the Eighth Joint National Committee (JNC 8). JAMA. 2014;311:507-20.

4. Torres Y, Aristizabal D, Montoya L. Diagnóstico del riesgo cardiovascular global, evaluación de su impacto poblacional, Medellín y sus corregimientos 2007-2008. Medellín 2008. Disponible en: http://www.medellin.gov.co/irj/go/km/docs/AlcaldiaMedellin/ SecretariaSalud/SitioTemporalSalud/descargables/Estudios Elnvestigaciones/Diagnostico \%20riesgo_\%20cardiovascular_Medellin_\%202007_2008.pdf.

5. Brundtland GH. From the World Health Organization. Reducing risks to health, promoting healthy life. JAMA. 2002;288:1974.

6. Hedayati SS, Elsayed EF, Reilly RF. Non-pharmacological aspects of blood pressure management: what are the data? Kidney Int. 2011;79:1061-70.

7. Group SR, Wright JT Jr, Williamson JD, Whelton PK, Snyder JK, Sink KM, et al. A randomized trial of intensive versus standard blood-pressure control. N Engl J Med. 2015;373:2103-16.

8. Group AS, Cushman WC, Evans GW, Byington RP, Goff DC Jr, Grimm RH Jr, et al. Effects of intensive blood-pressure control in type 2 diabetes mellitus. N Engl J Med. 2010;362:1575-85.

9. Atkins ER, Rodgers A. More versus less blood pressure lowering: an update. Clin Ther. 2016;38:2135-41.

10. Xie X, Atkins E, Lv J, Bennett A, Neal B, Ninomiya T, et al. Effects of intensive blood pressure lowering on cardiovascular and renal outcomes: updated systematic review and meta-analysis. Lancet. 2016;387(10017):435-43.

11. Agabiti Rosei E. Target blood pressure for treatment: should current recommendations be changed? Hypertension. 2016;68:263-5.

12. Wald DS, Law M, Morris JK, Bestwick JP, Wald NJ. Combination therapy versus monotherapy in reducing blood pressure: meta-analysis on 11,000 participants from 42 trials. Am J Med. 2009;122:290-300.

13. Mancia G, Grassi G. Aggressive blood pressure lowering is dangerous: the J-curve: pro side of the arguement. Hypertension. 2014;63:29-36.

14. Bress AP, Kramer H, Khatib R, Beddhu S, Cheung AK, Hess R, et al. Potential Deaths Averted and Serious Adverse Events Incurred From Adoption of the SPRINT (Systolic Blood Pressure Intervention Trial) Intensive Blood Pressure Regimen in the United States: Projections From NHANES (National Health and Nutrition Examination Survey). Circulation. 2017;135:1617-28. 